\title{
Growth hormone in heart failure revisited: An old story retold
}

\author{
Antonio Cittadini', Roberta D'Assante² \\ 1 Department of Translational Medical Sciences, Division of Internal Medicine \\ (Metabolic and Cardiac Rehabilitation Unit), "Federico II" University of Naples \\ 2 IRCCS SDN, Naples, Italy
}

\begin{abstract}
Heart failure (HF) is a disease characterized by increasing prevalence, huge direct and indirect costs, and an ominous prognosis, worse than many cancers. At the beginning of the $90 \mathrm{~s}$, growth hormone (GH) was proposed as potential adjunctive therapy in HF mostly due to its growth-promoting, vasodilating, and anti-apoptotic actions. However, although several uncontrolled clinical studies showed that GH therapy improved several cardiovascular parameters, two robust trials failed to confirm these findings. Dwelling upon potential explanations for such apparent discrepancy led to the hypothesis that HF patients exhibit an inhomogeneous basal activity of the $\mathrm{GH} /$ insulin-like growth factor 1 (IGF-1) axis, ranging from GH/IGF-1 deficiency to GH resistance. This complex phenomenon was then reconsidered in the context of the socalled multiple hormone deficiency syndrome (MHD), that is the recognition that HF is characterized not only by the hyperactivation of several signaling pathways including the adrenergic, the renin-angiotensin-aldosterone and cytokine systems, but also by a reduced anabolic drive leading to a state of anabolic/catabolic imbalance. Mounting evidence support the concept that such imbalance is not a mere epiphenomen, since it exerts a significant impact on clinical performance and more importantly, on survival. Therefore, the paradigm shift to reconsider HF as MHD represented the underpinning to implement clinical trials focused on hormone replacement therapies in congestive heart failure (CHF). With regard to $\mathrm{GH}$ replacement therapy, one controlled single-blind study yielded promising results, and we are currently conducting a double-blind controlled trial, as well a large Registry study to evaluate the impact of MHD on HF progression.
\end{abstract}

Corresponding author: Prof. Antonio Cittadini, Department of Translational Medical Sciences, Division of Internal Medicine (Metabolic and Cardiac Rehabilitation Unit), "Federico II" University of Naples, Via S. Pansini 5, 80131 Napoli, Italy. E-mail: antonio.cittadini@unina.it

Key words: Heart failure; hormones; anabolic deficiency; growth hormone; IGF-1; multiple hormone deficiency syndrome; TOSCA registry.

Received for publication: 13 August 2018

Accepted for publication: 27 August 2018

CCopyright A. Cittadini and R. D'Assante, 2018

Tipografia PI-ME Editrice, Italy

Monaldi Archives for Chest Disease 2018; 88:989

doi: 10.4081/monaldi.2018.989

This article is distributed under the terms of the Creative Commons Attribution Noncommercial License (by-nc 4.0) which permits any noncommercial use, distribution, and reproduction in any medium, provided the original author(s) and source are credited.

\section{Beginning of the story: Rationale for a growth factor approach to heart failure}

Several lines of experimental evidence support the concept that the activation of the growth hormone/IGF-1 axis exerts beneficial effects on the cardiovascular system. Growth hormone (GH) and IGF-1 induce a unique pattern of myocardial growth, characterized by unchanged capillary density, preserved diastolic function, and normal or augmented systolic function [1]. This has been shown in several rodent models, as well as in acromegalic patients with short disease duration, and in controls subjects undergoing GH therapy [2-6]. Of note, at gene level, there is no evidence of maladaptive reprogramming, with attendant changes of major calcium regulating proteins. In this concern, IGF-1 has been shown to acutely augment mammalian intrinsic contractility with a unique mechanism of calcium sensitization of the myofilaments [7]. Also in chronic models of GH/IGF-1 excess in rats, myocardial contractility is improved primarily due to an increase of myofilament responsiveness [8]. It should be mentioned that inotropic drugs acting as calcium sensitizers without changes in intracellular $\mathrm{Ca}^{2+}$ flux are eagerly searched for insofar as this mechanism is not associated with untoward effects such as arrhythmias. Activation of akt signaling, which lies downstream of IGF-1 receptor, appears to induce SERCA2 upregulation in several animal models, that in turn provides benefits in heart failure (HF) [9]. Furthermore, evidence has been accumulated pointing to a distinct role of IGF-1, the main effector of $\mathrm{GH}$ action, as a survival factor. Specifically, IGF-1 is endowed with peculiar cardioprotective functions mediated by the inhibition of cardiomyocytes apoptosis [10]. IGF-1 and its downstream cascade appears to play a significant role in the intricate framework of myocardial growth and survival activating PI3k/Akt signaling, as opposed by negative feedback controlled by several alternative pathways, including SOCSs [11]. Such observations prompted numerous studies that have consistently demonstrated beneficial effects of IGF-1 in the setting of experimental heart failure, using either exogenous administration or gene manipulation [12-14].

With regard to vascular physiology, IGF-1 provides for the tonic production of nitric oxide from the endothelial cells and for preserving endothelial function [15]. Because of the wide spectrum of atherogenic mechanisms that are inhibited by NO [16], IGF-1 acts as vasculoprotective and anti-atherogenic factor [17]. Indeed, subjects with chronically impaired IGF-1 production display endothelial dysfunction and undergo premature atherosclerosis and major vascular clinical events [18-20].

\section{Clinical trials}

Most animal studies have reported beneficial effects of enhanced myocardial GH/IGF-1 signaling, obtained with various approaches, from exogenous GH or IGF-1 administration, to gene therapy or myocardial IGF-1 overexpression [21-24]. A wide array of improved cardiovascular outcome measures was described including attenuated left ventricular 
remodeling, reduced apoptosis and wall stress, improved cardiac mechanics, and, most importantly, prolonged survival. However, at variance with experimental investigations, human trials have yielded conflicting results. The first study from Fazio's group demonstrated that recombinant human $\mathrm{GH}$ administered for three months to patients with idiopathic dilated cardiomyopathy increased myocardial mass and reduced the size of the left ventricular chamber, resulting in improvement in hemodynamics, myocardial energy metabolism, and clinical status [25]. Such findings were subsequently confirmed [26] and expanded by several independent groups, although employing uncontrolled study designs $[27,28]$. However, the interest toward GH therapy in heart failure tended to vanish when two robust double-blind placebocontrolled trials failed to confirm such findings [29,30].

\section{Pitfalls of GH trials in congestive heart failure: Lack of evaluation of basal GH/IGF-1 status}

What were the main reasons of such inconsistencies? An obvious answer is the lack of a placebo arm, that may clearly impair the study results by neutralizing the placebo effect. A second reason might be related to the relatively short duration of GH therapy: it is possible myocardial, skeletal, and ventilatory muscles may change their properties only after a longer exposure to GH/GG-1. Another argument that has been put forward are the questionable end-points employed in these trials. For instance, Osterziel and colleagues calculated the sample size using ejection fraction changes as primary end-point [29]. Apart from the criticism on ejection fraction as erroneously assumed measure of myocardial contractility [31], most clinical trials currently employ indexes of physical performance such as peak oxygen consumption, since an increase of systolic function may be associated with augmented oxygen demand, that may negatively reverberate on survival.

However, the greatest pitfall in GH human trials appears to be the lack of evaluation of the basal GH/IGF-1 status. This stems from the observation that when the same authors of the largest randomized controlled trial re-analyzed their findings, they found a correlation between the changes in IGF-1 and those in LV mass, and a significant increase of ejection fraction in those patients displaying $\mathrm{GH}$-induced increases of IGF-1 of more than the median value of $80 \mathrm{pg} / \mathrm{mL}$ [32]. Similar conclusions were reached in a meta-analysis by Le Corvoisier and colleagues [33]. Thus, the hypothesis has been put forward that since $\mathrm{HF}$ patients display a large variability of GH/IGF-1 activity, ranging from an intact axis to $\mathrm{GH} / \mathrm{IGF}-1$ deficiency to $\mathrm{GH}$ resistance, it is reasonable to expect different responses in IGF-1 production following $\mathrm{GH}$ administration, and, consequently, a high variability of the selected clinical outcomes. Indeed, several studies have clearly demonstrated that patients with HF may display an intact GH/IGF-1 axis, reduced IGF-1 circulating levels, $\mathrm{GH}$ deficiency or conditions of $\mathrm{GH}$ resistance, particularly in advanced stages of the disease, characterized by concomitant high GH and low IGF-1 serum levels [34,35]. While treating patients with GH/IGF-1 deficiency may in theory improve cardiovascular status, it is reasonable to speculate that replacement or even higher $\mathrm{GH}$ doses may yield subtle or neutral effects in patients with an intact axis and particularly in those with $\mathrm{GH}$ resistance.

\section{Paradigm shift: HF as a multiple hormonal deficiency syndrome}

The observation that a subset of HF patients is affected with GH/IGF-1 deficiency may be viewed in a larger perspective, i.e. the presence of hormonal comorbidities, particularly those involving the anabolic drive.
There is an active research area aimed at identifying and treating relevant comorbidities in HF that may affect its progression. Non-cardiac comorbidities playing a significant role include chronic obstructive pulmonary disease, renal dysfunction, arthritis, cognitive dysfunction and depression, and anemia/iron deficiency [36]. As a prototype, treating a subset of patients with coexisting HF and iron deficiency or anemia with iron therapy or epoietin [37,38] may confer beneficial effects, as in other non-cardiac conditions [39]. In this context, mounting evidence support the concept that multiple anabolic deficiencies are common in chronic HF and identify subgroups of patients with higher mortality [40]. The first evidence concerning the role of anabolic deficiencies in HF patients comes from a landmark study by Jankowska et al. [40]; specifically, deficit of each anabolic axis [adrenal, gonadal and somatotropic axes) represents an independent marker of poor outcome in HF patients and the coexistence of more than one deficiency identifies a subgroup of patients with a higher mortality. The most involved hormonal axes include $\mathrm{GH}$, its tissue effector IGF-1, thyroid hormone, insulin and anabolic steroids. Taken together, these alterations could be recognized as multiple hormone deficiency syndrome (MHD) in HF patients [41]. MHD bears a significant impact on cardiac performance, clinical status, HF progression and survival [41-43]. In other words, despite the effectiveness of the neurohormonal model to explain disease progression and the many insights that provided the basis for the development of new therapies, such model clearly fails to fully explain disease progression. MHD may represent a complementary model of HF progression, based on the observation that $\mathrm{HF}$ is not only characterized by excessive stimulation of the adrenergic, the renin-angiotensin-aldosterone, and the cytokine system, but also by the reduced activity of the principal anabolic axes of the human body, thus leading to an anabolic/catabolic imbalance [44].

\section{GH deficiency in heart failure}

Chief among these is the reduced activity of the GH/IGF-1 axis. Low levels of IGF-1 correlate with the degree of systolic dysfunction, the presence of cachexia and skeletal muscle weakness, and indices of neurohormonal and cytokine activation [45]. Several independent groups have documented low IGF-1 levels in congestive heart failure (CHF). Broglio et al. reported low IGF-1 levels in well-nourished patients with severe $\mathrm{LV}$ dysfunction, as well as blunted response to $\mathrm{GHRH}$, both alone or combined with arginine [46,47]. This finding was subsequently confirmed by Anwar et al. in an elderly population of patients hospitalized for HF [48] and by Kontoleon et al. in 23 ambulatory patients with stable HF [49]. Anker and co-workers did not find reduced IGF-1 levels in non-cachectic patients with HF, while in cachectic patients circulating IGF-1 was significantly decreased compared with controls [50]. These authors also provided evidence for $\mathrm{GH}$ resistance, defined as high GH levels and low IGF-1 levels, in $60-70 \%$ of cachectic and $20-30 \%$ of non-cachectic patients with HF. In 158 consecutive patients with $\mathrm{HF}$, we recently found the prevalence of $\mathrm{GH}$ deficiency to be $40 \%$ [51,52]. This finding is not unexpected considering that Broglio and coworkers reported a similar prevalence of GH deficiency [47] and that Jankowska et al. have recently reported an average prevalence of $64 \%$ of IGF-1 deficiency in 208 patients with HF NYHA class I-IV [40].

Apart from HF, GH deficiency increases cardiovascular mortality. Although data regarding the mortality rates of patients with $\mathrm{GH}$ deficiency are limited, an increased mortality rate among hypopituitary patients compared with the general population has been clearly documented. The first report dates back to 1990 [53], and it has been confirmed subsequently by several independent groups [54 55]. Cardiovascular disease has been suggested as a primary cause of death, whereas cancer statistics might be influenced by the number of malig- 
nancies causing the pituitary disease. To support the concept that the pathophysiological link between increased mortality and hypopituitarism is GH deficiency, two studies showed a normal mortality rate in GH deficient patients treated with $\mathrm{GH}$ compared with the general population [57]. On the other hand, it has been suggested that hypopituitarism affects several cardiac diseases including Takotsubo cardiomyopathy $[57,58]$.

The pivotal role of low IGF-1 levels in determining future cardiovascular problems is further demonstrated by large population-based studies. In fact, in the general population low IGF-1 levels predict the development of ischemic heart disease, HF, and cardiovascular mortality [59-61].

Taken together, low IGF-1 levels are associated with increased mortality in the normal population and in $\mathrm{GH}$ deficient humans. On the other hand, low IGF-1 levels are commonly found in HF. Therefore, it is reasonable to expect a worse prognosis in patients with coexisting $\mathrm{HF}$ and low IGF-1 levels. This bring us back to the study of Jankowska and colleagues, showing that multiple anabolic deficiencies, including IGF1 levels below the tenth percentile of healthy peers, increase mortality in a population of CHF patients [40], and by Petretta et al., that also demonstrated that a low IGF-1/GH ratio and high NT-proBNP levels are independent predictors of death in HF patients without cachexia [62], and more recently by Arcopinto and colleagues [63]. Therefore, it appears that the finding of low IGF-1 circulating levels in CHF is not an epiphenomenon or a mere biochemical marker, but is mechanistically linked to CHF clinical status and to disease progression.

From this background, IGF-1 emerges as a molecule endowed with potential beneficial effects on the cardiovascular system, and its reduced production increases mortality in normal individuals and, in particular, in patients with chronic HF.

\section{Ongoing studies}

Given this solid background, several clinical studies were implemented to evaluate the effects of $\mathrm{GH}$ replacement therapy in patients with coexisting $\mathrm{GH}$ deficiency and $\mathrm{CHF}$. In a randomized, single-blind, controlled trial, one hundred fifty-eight patients with CHF, New York Heart Association class II-IV, underwent a GH stimulation test [51]. Interestingly, as many as $40 \%$ of patients with $\mathrm{CHF}$ were $\mathrm{GH}$ deficient. Moreover, compared to $\mathrm{GH}$ deficient patients, $\mathrm{GH}$ sufficient patients showed smaller end-diastolic and end-systolic LV volumes, lower LV endsystolic wall stress, higher RV performance, lower estimated systolic pulmonary artery pressure, higher peak $\mathrm{VO}_{2}$ and increased ventilatory efficiency. GH deficiency was also associated with increased all-cause mortality [52]. Sixty-three patients satisfied the criteria for GH deficiency, and 56 of them were enrolled in the pharmacological trial. The treated group received $\mathrm{GH}$ at a replacement dose of $0.012 \mathrm{mg} / \mathrm{kg}$ every second day (2.5 IU). GH replacement therapy in these patients improves exercise capacity, vascular reactivity, left ventricular function, and indices of quality of life [51]. After 4 years of follow-up, such beneficial actions not only were sustained, but improved consistently [64].

A major limitation of these trials was the lack of a placebo arm, and, accordingly, a double-blind-placebo controlled study is currently ongoing in our Department, sponsored by the GGI 2016 award (https://www.grantforgrowthinnovation.org/en/ggi2016/winners.html). Specifically, the objective of the study is to determine whether treatment of GH deficiency in patients with chronic HF improves peak oxygen consumption, a recognized surrogate end-point of HF progression, and several secondary end-points including NT-proBNP, measures of left ventricular architecture and function, quality of life, and anxiety and depression scores.
Another major ongoing study in this field is the T.O.S.CA. (Trattamento Ormonale nello Scompenso CArdiaco; Hormone Therapy in Heart Failure) Registry (clinicaltrial.gov: NCT02335801) which tests the hypothesis that anabolic deficiencies reduce survival in a large population of mild-to-moderate CHF patients. The T.O.S.CA. Registry is a prospective multicenter observational study coordinated by the "Federico II" University of Naples, and involves 19 centers situated throughout Italy. Thyroid hormones, insulin-like growth factor-1, total testosterone, dehydropianoandrosterone and insulin are measured at baseline and every year for a patient-average follow-up of 3 years. Subjects with chronic HF are divided into two groups: patients with 1 or no anabolic deficiency, and patients with 2 or more anabolic deficiencies at baseline. The primary endpoint is the composite of all-cause mortality and cardiovascular hospitalization. Secondary endpoints include the composite of all-cause mortality and hospitalization, the composite of cardiovascular mortality and cardiovascular hospitalization, and change of peak oxygen consumption. Patient enrollment started in April 2013, and was completed in July 2017. Demographics and main clinical characteristics of enrolled patients are provided in this article. Detailed cross-sectional results will be available in late 2018 [65].

The T.O.S.CA Registry represents the most robust prospective observational trial on MHD in the field of HF. The study findings will advance our knowledge with regard to the intimate mechanisms of HF progression and hopefully pave the way for future randomized clinical trials of single or multiple hormonal replacement therapies in CHF.

\section{Conclusions}

GH therapy in HF looks like an old story retold. Although the rationale for its use is still solid, the choice of the right population to treat turned out to be wrong. Not all HF patients will probably benefit from a growth factor approach, but only the subset of subjects displaying coexisting GH deficiency and HF. This approach is currently viewed in the context of the larger scenario of HF comorbidities, and in particular, of hormone deficiencies. Whether MHD is a mere cluster of biochemical hormone abnormalities or a strong independent predictor of $\mathrm{HF}$ progression is still unknown. Ongoing studies will soon provide a definitive answer to these outstanding questions.

\section{References}

1. Saccà L. Growth hormone therapy for heart failure: swimming against the stream. J Card Fail 1999;5:269-75.

2. Cittadini A, Mantzoros CS, Hampton TG, et al. Differential cardiac effects of growth hormone and insulin-like growth factor-1 in the rat. A combined in vivo and in vitro evaluation. Circulation 1999;100:2177-83.

3. Cittadini A, Berggren A, Longobardi S, et al. Supraphysiological doses of $\mathrm{GH}$ induce rapid changes in cardiac morphology and function. J Clin Endocrinol Metab 2002;87:1654-9.

4. Fazio S, Cittadini A, Biondi B, et al. Cardiovascular effects of shortterm growth hormone hypersecretion. J Clin Endocrinol Metab 2000;85:179-82.

5. Mosca S, Paolillo S, Colao A, et al. Cardiovascular involvement in patients affected by acromegaly: An appraisal. Int $\mathrm{J}$ Cardiol 2013;167:1712-8.

6. Giannoulis MG, Boroujerdi MA, Powrie J, et al. Gender differences in growth hormone response to exercise before and after rhGH administration and the effect of rhGH on the hormone profile of fit normal adults. Clin Endocrinol (Oxf) 2005;62:315-22. 
7. Cittadini A, Ishiguro Y, Strömer H, et al. Insulin-like growth factor1 but not growth hormone augments mammalian myocardial contractility by sensitizing the myofilament to $\mathrm{Ca} 2+$ through a wortmannin-sensitive pathway: studies in rat and ferret isolated muscles. Circ Res 1998;83:50-9.

8. Strömer H, Cittadini A, Douglas PS, et al. Exogenously administered growth hormone and insulin-like growth factor-I alter intracellular $\mathrm{Ca} 2+$ handling and enhance cardiac performance. In vitro evaluation in the isolated isovolumic buffer-perfused rat heart. Circ Res 1996;79:227-36.

9. Cittadini A, Monti MG, Iaccarino G, et al. Adenoviral gene transfer of Akt enhances myocardial contractility and intracellular calcium handling. Gene Ther 2006;13:8-19.

10. Saetrum Opgaard 0, Wang PH. IGF-I is a matter of heart. Growth Horm IGF Res 2005;15:89-94.

11. Cittadini A, Monti MG, Iaccarino G, et al. SOCS1 gene transfer accelerates the transition to heart failure through the inhibition of the gp130/JAK/STAT pathway. Cardiovasc Res 2012;96:381-90.

12. Cittadini A, Isgaard J, Monti MG, et al. Growth hormone prolongs survival in experimental postinfarction heart failure. J Am Coll Cardiol 2003;41:2154-63.

13. Li Q, Li B, Wang X, Leri A, et al. Overexpression of insulin-like growth factor-1 in mice protects from myocyte death after infarction, attenuating ventricular dilation, wall stress, and cardiac hypertrophy. J Clin Invest 1997;100:1991-9.

14. Kusano K, Tsutsumi Y, Dean J, et al. Long-term stable expression of human growth hormone by $\mathrm{rAAV}$ promotes myocardial protection post-myocardial infarction. J Mol Cell Cardiol 2007;42:390-9.

15. Repetto S, Salani B, Maggi D, et al. Insulin and IGF-I phosphorylate eNOS in HUVECs by a caveolin-1 mechanism. Biochem Biophys Res Com 2005;337:849-52.

16. Griendling KK, FitzGerald GA. Oxidative stress and cardiovascular injury: Part II: animal and human studies. Circulation 2003 108:2034-40.

17. Cittadini A, Monti MG, Castiello MC, et al. Insulin-like growth factor-1 protects from vascular stenosis and accelerates reendothelialization in a rat model of carotid artery injury. J Thromb Haemost 2009;7:1920-8.

18. Saccà L, Cittadini A, Fazio S. Growth hormone and the heart. Endocr Rev 1994;15:555-73.

19. Rosen T, Eden S, Larson G, et al. Cardiovascular risk factors in adult patients with growth hormone deficiency. Acta Endocrinol 1993;129:195-200.

20. Lanes R, Soros A, Flores K, et al. Endothelial function, carotid artery intima-media thickness, epicardial adipose tissue, and left ventricular mass and function in growth hormone-deficient adolescents: apparent effects of growth hormone treatment on these parameters. J Clin Endocr Metab 2005;90:3978-82.

21. Duerr RL, Huang S, Miraliakbar HR, et al. Insulin-like growth factor-1 enhances ventricular hypertrophy and function during the onset of experimental cardiac failure. J Clin Invest 1995;95:619-27.

22. Cittadini A, Grossman JD, Napoli R, et al. Growth hormone attenuates early left ventricular remodeling and improves cardiac function in rats with large myocardial infarction. J Am Coll Cardiol 1997;29:1109-16.

23. Cittadini A, Grossman JD, Stromer H, et al. Importance of an intact growth hormone/insuline-like growth factor 1 axis for normal postinfarction healing: Studies in dwarf rats. Endocrinology 2001;142:332-8.

24. Kusano K, Tsutsumi Y, Dean J, et al. Long-term stable expression of human growth hormone by rAAV promotes myocardial protection post-myocardial infarction. J Mol Cell Cardiol 2007;42:390-9.

25. Fazio S, Sabatini D, Capaldo B, et al. A preliminary study of growth hormone in the treatment of dilated cardiomyopathy. N Engl J Med 1996;334:809-14.

26. Fazio S, Palmieri EA, Affuso F, et al. Effects of growth hormone on exercise capacity and cardiopulmonary performance in patients with chronic heart failure. J Clin Endocrinol Metab 2007;92:4218-23.

27. Genth-Zotz S, Zotz R, Geil S, et al. Recombinant growth hormone therapy in patients with ischemic cardiomyopathy: effects on hemodynamics, left ventricular function, and cardiopulmonary exercise capacity. Circulation 1999;99:18-21.

28. Adamopoulos S, Parissis JT, Paraskevaidis I, et al. Effects of growth hormone on circulating cytokine network, and left ventricular contractile performance and geometry in patients with idiopathic dilated cardiomyopathy. Eur Heart J 2003;24:2186-96.

29. Osterziel KJ, Strohm 0, Schuler J, et al. Randomised, double-blind, placebo-controlled trial of human recombinant growth hormone in patients with chronic heart failure due to dilated cardiomyopathy. Lancet 1998;351:1233-7.

30. Isgaard J, Bergh $\mathrm{CH}$, Caidahl $\mathrm{K}$, et al. A placebo-controlled study of growth hormone in patients with congestive heart failure. Eur Heart J 1998;19:1704-11.

31. Konstam MA, Abboud FM. Ejection fraction: Misunderstood and overrated (changing the paradigm in categorizing heart failure). Circulation 2017;135:717-9

32. Perrot A, Ranke MB, Dietz R, et al. Growth hormone treatment in dilated cardiomyopathy. J Card Surg. 2001;16:127-31.

33. Le Corvoisier P, Hittinger L, Chanson P, et al. Cardiac effects of growth hormone treatment in chronic heart failure: A metaanalysis. J Clin Endocrinol Metab 2007;92:180-5.

34. Anker SD, Volterrani M, Pflaum CD, et al. Acquired growth hormone resistance in patients with chronic heart failure: implications for therapy with growth hormone. J Am Coll Cardiol 2001;38:443-52.

35. Arcopinto M, Salzano A, Isgaard J, et al. Hormone replacement therapy in heart failure. Curr Opin Cardiol 2015;30:277-84.

36. Lang CC, Mancini DM. Non-cardiac comorbidities in chronic heart failure. Heart 2007;93:665-71.

37. Kang CK, Pope M, Lang CC, et al. Iron deficiency in heart failure: Efficacy and safety of intravenous iron therapy. Cardiovasc Ther $2017 ; 35$.

38. Vullaganti S, Goldsmith J, Teruya S, et al. Cardiovascular effects of hemoglobin response in patients receiving epoetin alfa and oral iron in heart failure with a preserved ejection fraction. J Geriatr Cardiol 2014;11:100-5.

39. Saccà F, Piro R, De Michele G, et al. Epoetin alfa increases frataxin production in Friedreich's ataxia without affecting hematocrit. Mov Disord 2011;26:739-42.

40. Jankowska EA, Biel B, Majda J, et al. Anabolic deficiency in men with chronic heart failure. Prevalence and detrimental impact on survival. Circulation 2006;114:1829-37.

41. Saccà L. Heart failure as a multiple hormonal deficiency syndrome. Circ Heart Fail 2009;2:151-6.

42. Arcopinto M, Salzano A, Bossone E, et al. Multiple hormone deficiencies in chronic heart failure. Int J Cardiol 2015;184:421-3.

43. Salzano A, Marra AM, Ferrara F, et al. Multiple hormone deficiency syndrome in heart failure with preserved ejection fraction. Int J Cardiol 2016;225:1-3.

44. Arcopinto M, Cittadini A. Hormonal alterations in heart failure: anabolic impairment in chronic heart failure - diagnostic, prognostic and therapeutic issues. Front Horm Res 2014;43:57-69.

45. Niebauer J, Pflaum CD, Clark AL, et al. Deficient insulin-like growth factor I in chronic heart failure predicts altered body composition, anabolic deficiency, cytokine and neurohormonal activation. J Am Coll Cardiol 1998;32:393-7. 
46. Broglio F, Fubini A, Morello M, et al. Activity of GH/IGF-I in patients with dilated cardiomyopathy. Clin Endocrinol 1999;50:417-30.

47. Broglio F, Benso A, Gottero C, et al. Patients with dilated cardiomyopathy show reduction of the somatotrophic responsiveness to GHRH both alone and combined with arginine. Eur J Endocrinol 2000;142:157-63.

48. Anwar A, Gaspoz JM, Pampallona S, et al. Effect of congestive heart failure on the insulin-like growth factor-1 system. Am J Cardiol 2002;90:1402-5.

49. Kontoleon PE, Anastasiou-Nana MI, Papapetrou PD, et al. Hormonal profile in patients with congestive heart failure. Int J Cardiol 2003;87:179-83.

50. Anker SD, Volterrani M, Pflaum CD, et al. Acquired growth hormone resistance in patients with chronic heart failure: implications for therapy with growth hormone. J Am Coll Cardiol 2001;38:443-52.

51. Cittadini A, Saldamarco L, Marra AM, et al. Growth hormone deficiency in patients with chronic heart failure and beneficial effects of its correction. J Clin Endocrinol Metab 2009;94:3329-36.

52. Arcopinto M, Salzano A, Giallauria F, et al. Growth hormone deficiency is associated with worse cardiac function, physical performance, and outcome in chronic heart failure: insights from the T.O.S.CA. GHD study. PLoS One 2017;12:e0170058.

53. Rosén T, Bengtsson BA. Premature mortality due to cardiovascular disease in hypopituitarism. Lancet 1990; 336:285-8.

54. Bulow B, Hagmar L, Mikoczy Z, et al. Increased crebrovascular mortality in patients with hypopituitarism. Clin Endocrinol (0xf) 1997;46:75-81.

55. Tomlinson JW, Holden N, Hills RK, et al. Association between premature mortality and hypopituitarism. West Midlands Prospective Hypopituitary Study Group. Lancet 2001;357:425-31.

56. Stochholm K, Christiansen J, Laursen T, et al. Mortality and reduced growth hormone secretion Horm Res 2007;68:173-6.
57. Plácido R, Martins AF, Robalo Martins S, et al. Takotsubo Syndrome: A pathway through the pituitary disease. Case Rep Cardiol 2016;2016:9219018.

58. Bossone E, Lyon A, Citro R, et al. Takotsubo cardiomyopathy: An integrated multi-imaging approach. Eur Heart $\mathrm{J}$ Cardiovasc Imaging 2014;15:366-77.

59. Juul A, Scheike T, Davidsen M, et al. Low serum insulin-like growth factor I is associated with increased risk of ischemic heart disease: a population-based case-control study. Circulation 2002;106:939-44.

60. Vasan RS, Sullivan LM, D’Agostino RB, et al. Serum insulin-like growth factor I and risk for heart failure in elderly individuals without a previous myocardial infarction: the Framingham Heart Study. Ann Intern Med 2003;139:642-8.

61. Laughlin GA, Barrett-Connor E, Criqui MH, et al. The prospective association of serum insulin-like growth factor I (IGF-I) and IGFbinding protein-1 levels with all cause and cardiovascular disease mortality in older adults: the Rancho Bernardo Study. J Clin Endocrinol Metab 2004;89:114-20.

62. Petretta M, Colao A, Sardu C, et al. NT-proBNP, IGF-I and survival in patients with chronic heart failure. Growth Horm IGF Res 2007; 17:288-96.

63. Cittadini A, Marra AM, Arcopinto M, et al. Growth hormone replacement delays the progression of chronic heart failure combined with growth hormone deficiency: An extension of a randomized controlled single-blind study. JACC Heart Fail 2013;1:325-30.

64. Arcopinto M, Isgaard J, Marra AM, et al. IGF-1 predicts survival in chronic heart failure. Insights from the T.O.S.CA. (Trattamento Ormonale Nello Scompenso CArdiaco) registry. Int J Cardiol 2014;176:1006-8.

65. Bossone E, Arcopinto M, Iacoviello M, et al. Multiple hormonal and metabolic deficiency syndrome in chronic heart failure: rationale, design, and demographic characteristics of the T.O.S.CA. Registry. Intern Emerg Med 2018;13:661-71. 